\title{
The Correlation between Insertion Depth of Prodisc-C Artificial Disc and Postoperative Kyphotic Deformity - Clinical Importance of Insertion Depth of Artificial Disc -
}

\author{
Do-Youl Lee, Se-Hoon Kim, Jung-Keun Suh, Tai-Hyoung Cho, Yong-Gu Chung \\ Department of Neurosurgery, Korea University College of Medicine, Seoul, Korea
}

\begin{abstract}
Objective: This study was designed to investigate the correlation between insertion depth of artificial disc and postoperative kyphotic deformity after Prodisc- $C$ total disc replacement surgery, and the range of artificial disc insertion depth which is effective in preventing postoperative whole cervical or segmental kyphotic deformity.

Methods: A retrospective radiological analysis was performed in 50 patients who had undergone single level total disc replacement surgery. Records were reviewed to obtain demographic data. Preoperative and postoperative radiographs were assessed to determine C2-7 Cobb's angle and segmental angle and to investigate postoperative kyphotic deformity. A formula was introduced to calculate insertion depth of Prodisc-C artificial disc. Statistical analysis was performed to search the correlation between insertion depth of Prodisc- $\mathrm{C}$ artificial disc and postoperative kyphotic deformity, and to estimate insertion depth ofProdisc-C artificial disc to prevent postoperative kyphotic deformity.

Results: In this study no significant statistical correlation was observed between insertion depth of Prodisc- $\mathrm{C}$ artificial disc and postoperative kyphotic deformity regarding C2-7 Cobb's angle. Statistical correlation between insertion depth of Prodisc-C artificial disc and postoperative kyphotic deformity was observed regarding segmental angle $(p<0.05)$. It failed to estimate proper insertion depth of Prodisc-C artificial disc effective in preventing postoperative kyphotic deformity.

Conclusion: Postoperative segmental kyphotic deformity is associated with insertion depth of Prodisc-C artificial disc. Anterior located artificial disc leads to lordotic segmental angle and posterior located artificial disc leads to kyphotic segmental angle postoperatively. But C2-7 Cobb's angle is not affected by artificial disc location after the surgery.
\end{abstract}

Key Words: Total disc replacement • Postoperative kyphotic deformity • Insertion depth • Prodisc-C artificial disc

\section{INTRODUCTION}

Cervical total disc replacement (TDR) has been one of the most commonly performed procedures for the treatment of cervical radiculopathy since Dr. Goffin implanted Bryan (Medtronic Sofamor Danek, Memphis, TN, USA) artificial disc for the first time in January 2000. And it has been thought as an alternative method to traditional anterior cervical discectomy and fusion (ACDF).

TDR has some advantages compared with $\mathrm{ACDF}^{1,8)}$. It maintains physiologic range of cervical motion and the mecha-

- Received: April 13, 2012 - Revised: September 16, 2012

- Accepted: September 22, 2012

Corresponding Author: Se-Hoon Kim, MD

Department of Neurosurgery, Korea University Ansan Hospital, 516

Gojan 1-dong, Danwon-gu, Ansan 425-707, Korea

Tel: +82-31-412-5053, Fax: + 82-31-412-5054

Email: sean1050@gmail.com nical characteristics of functional spinal unit. TDR especially has been known to prevent or decrease the risk of adjacent segment degeneration.

There are, however, several complications in cervical TDR including postoperative kyphotic deformity.

Some causes have been suspected to lead to postopera tivekyphotic deformity. Yoon et al. ${ }^{14)}$ suggested over-milling at the dorsal endplate, artificial disc insertion angle, structural absence of lordosis in Bryan artificial discprosthesis, removal of posterior longitudinal ligament, pre-existing kyphosis. Yi et al. ${ }^{13)}$ proposed the correlation between insertion depth of the Bryan artificial disc prosthesis and postoperative kyphotic deformity.

The purpose of this study is to investigate the correlation between insertion depth of Prodisc-C (Synthes Inc., West Chester, PA, USA) artificial disc and postoperative kyphotic deformity after TDR surgery, and the range of artificial disc insertion depth to prevent postoperative whole cervical or segmental kyphotic deformity. 


\section{MATERIALS AND METHODS}

A retrospective analysis of medical record and radiogra phsof 50 patients was performed. They admitted to Korea University Anam Hospital, Seoul, Korea, and underwent on a single-level cervical TDR surgery with Prodisc-C artificial disc between July 2004 and June 2011.

Before the surgery, all patients were evaluated using cervical X-ray and magnetic resonance imaging. C2-7 Cobb's angles, segmental angles of the operative level with static neutral lateral X-ray were measured to evaluate pre- and post-operative final cervical sagittal balance and to investigate the postoperative kyphotic deformity.

C2-7 Cobb's angles were measured with the standard Cobb's angle method between the inferior margin of $\mathrm{C} 2$ and $\mathrm{C} 7$ vertebral bodies in a neutral position (Fig. 1). Segmental angles of the operative level were measured between the superior margin of upper cervical vertebral body and the inferior margin of lower cervical vertebral body (Fig. 2). A negative value means lordosis of cervical spine and a positive value means kyphosis.

Insertion depth of Prodisc-C artificial disc was calculated by a formula suggested by $\mathrm{Yi}$ et al. ${ }^{13)}$ as shown below. The length from the line connecting the posterior corner of superior and inferior vertebral bodies to the line connecting anterior corner of superior and inferior vertebral bodies at the operated disc level was measured. Then the length from the posterior body line to the center of the Prodisc- $\mathrm{C}$ artificial disc on the lateral cervical X-ray was measured (Fig. 3). But in this study, a modification was applied to the formula. $50 \%$ sub-

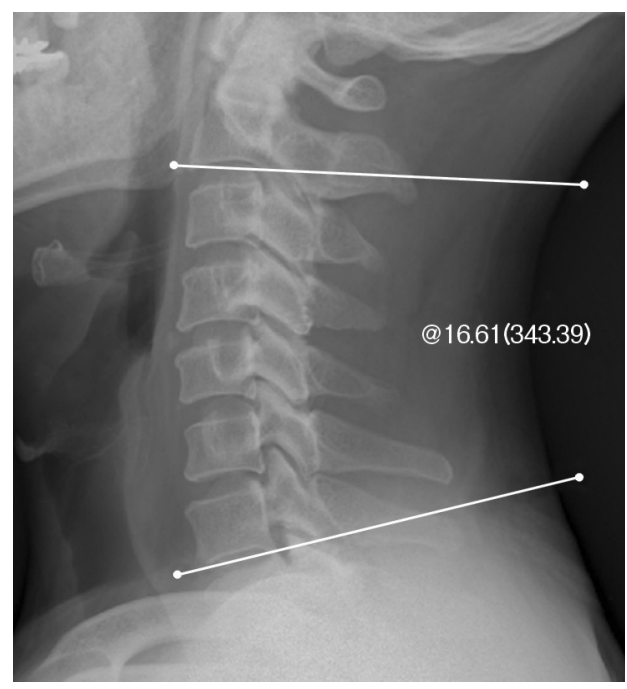

Fig. 1. C2-7 Cobb's angle. traction was not performed to estimate the insertion depth of Prodisc-C artificial disc from posterior vertebral margin. An insertion depth value of Prodisc-C artificial disc larger than $50 \%$ indicates that the disc location is anterior to the center of vertebral body and a smaller value than $50 \%$ indicates the location is posterior to the center of vertebral body.

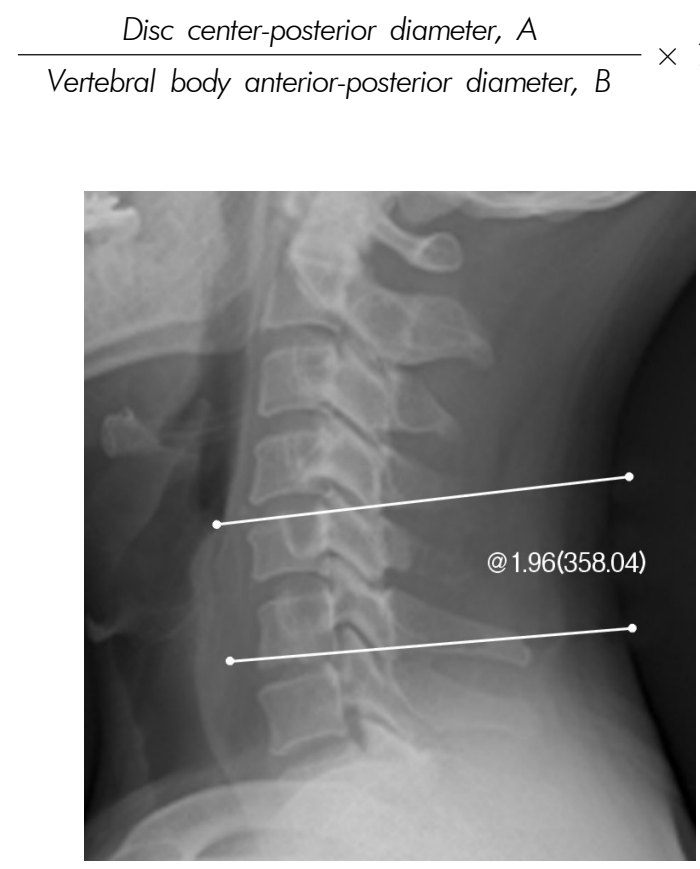

Fig. 2. Segmental angle.

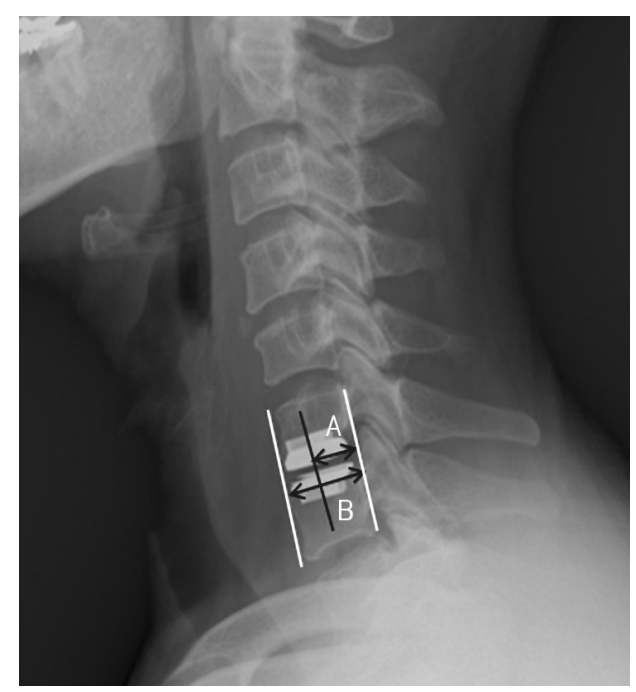

Fig. 3. Insertion depth of an Prodisc- $C$ artificial disc. " $A$ " indicates the length from the posterior body line to the center of the Prodisc- $\mathrm{C}$ artificial disc. " $\mathrm{B}$ " indicates the length from the line connecting the posterior corner of superior and inferior vertebral bodies to the line connecting anteriorcorner of superior and inferior vertebral bodies at the operated disc level. 
There are many different reports to define normal cervical lordosis and postoperative kyphotic deformity. With each of the definitions, we tried to find out any statistically significant correlation between artificial disc insertion depth and postoperative kyphotic deformity.

At first, we introduced the definitions that Heary and Karimi ${ }^{4)}$ proposed. Postoperative kyphotic deformity was defined as a loss of the normal lordotic cervical curvature of at least 5 degrees per motion segment (C2-7). With reference to this definition, the patients were divided into 2 groups as those with postoperative kyphotic deformity or not. And their insertion depths of Prodisc-C artificial disc were analyzedstatistically to find difference of mean value.

Then we used the definition that Gore et al. ${ }^{3)}$ proposed as normal cervical lordosis. In men, C2-7 Cobb's angle more than $22^{\circ}$ was defined as 'hyperlordotic', C2-7 Cobb's angle less than $16^{\circ}$ was defined as 'kyphotic' and that between $16^{\circ}$ and $22^{\circ}$ was defined as 'lordotic'. And in women, normal C2-7 Cobb's angle was from $15^{\circ}$ to $25^{\circ}$, C2-7 Cobb's angle more than $25^{\circ}$ was defined as 'hyperlordotic', and C2-7 Cobb's angle less than $15^{\circ}$ was defined as 'kyphotic'. When a patient's postoperative C2-7 Cobb's angle changed from 'hyperlordotic' to 'lordotic', 'hyperlordotic' to 'kyphotic' or 'lordotic' to 'kyphotic', we hypothesized that the patient showed postoperative kyphotic deformity. We analyzed those with postoperative kyphotic deformity to find out any difference from others.

But the methods using the definitions that Gore et al..$^{3)}$ pro posed can't distinguish those whose postoperative C2-7 Cobb's angle changes within the ranges of normal lordotic curve. So other concept of postoperative kyphotic deformity was introduced. When a patient'spostoperative C2-7 Cobb's angle decreased more than 5\% of preoperative C2-7 Cobb's angle, he/she was hypothesized to show postoperative kyphotic deformity and analyzed to search any correlation with artificial disc insertion depth.

Finally, pre- and post-operative segmental angles were evaluated. When a patient's postoperative segmental angle of operative level decreased more than $5 \%$ of preoperative one, we hypothesized that the patient showed postoperative kyphotic deformity. On the contrary, when postoperative segmental angle of operative level increased more than 5\%, we assumed that the patient showed postoperative lordotic change. When the range of changes was within $5 \%$ of preoperative segmental angle of operative level, we hypothesized that the patient didn't show any deformity. These 3 groups were analyzed statistically with insertion depths of Prodisc-C artificial disc using ANOVA test.

Pre- and post-operative C2-7 Cobb's angles and segmental angles of operative level were measured with quantitative measurement analysis software, PACS workstation (Centricity 2.0;
General Electric Medical Systems, Milwau- kee, WI, USA). This program uses extrapolative algorithms to calculate the intersecting angle between 2 lines drawn by the investigator.

\section{Statistical analysis}

Statistical analysis was performed to search the correlation between insertion depth of Prodisc-C artificial disc and postoperative kyphotic deformity, and to estimate insertion depth of Prodisc-C artificial disc to prevent postoperative kyphotic deformity. Analysis of variance (ANOVA) test with SPSS software for Windows (version 20.0; SPSS Inc., Chicago, IL, USA) was performed.

\section{RESULTS}

Patients consisted of 31 men and 19 women, a ratio of 1.63: 1. Mean age was 48.84 years (range, $26-77$ years). The mean duration of follow-up was 19.2 months (range, 9-28 months). Preoperative diagnosis included cervical herniated nucleus pulposus (41 patients), cervical spondylosis (8 patients), and foraminal stenosis (1 patient). The operated levels are as the following: C3/4 (3 patients), C4/5 (7 patients), C5/6 (29 patients), C6/7 (11 patients) (Table 1).

Mean disc insertion depth was 53.98\%, ranging from 42.41 to $67.63 \%$. An insertion depth value of Prodisc-C artificial disc larger than $50 \%$ indicates that the disc location is anterior to the center of vertebral body, and a smaller value than $50 \%$ indicates the location is posterior to the center of vertebral body, as mentioned above.

According to the definition that Heary and Karimi ${ }^{4)}$ proposed, fifteen patients were classified at the group with postoperative kyphotic deformity. And their mean disc insertion depth was 53.33\%. But the mean disc insertion depth of the

Table 1. Demographics

\begin{tabular}{ll}
\hline \hline Variable & Value \\
\hline Number of patients & 50 \\
Mean age (years) & 48.84 (range, 26-77) \\
Male/female & $31 / 19$ \\
Operation level & \\
C3/4 & 3 \\
C4/5 & 7 \\
C5/6 & 29 \\
C6/7 & 11 \\
Diagnosis & \\
Cervical HNP & 41 \\
Cervical spondylosis & 8 \\
Foraminal stenosis & 1 \\
\hline
\end{tabular}

HNP: herniated nucleus pulposus 
Table 2. Mean disc insertion depth between the group with postoperative kyphotic deformity and the group without deformity

\begin{tabular}{ccc}
\hline \hline $\begin{array}{c}\text { Definitions of kyphotic deformity } \\
\text { in C2-7 Cobb's angle }\end{array}$ & $\begin{array}{c}\text { Group with postoperative } \\
\text { kyphotic deformity }\end{array}$ & $\begin{array}{c}\text { Group without postoperative } \\
\text { kyphotic deformity }\end{array}$ \\
\hline Decrease more than 5 degrees & $53.33 \%(n=15)$ & $54.26 \%(n=35)$ \\
Change of groups & $53.07 \%(n=16)$ & $54.41 \%(n=34)$ \\
Decrease more than 5\% & $53.36 \%(n=26)$ & $54.65 \%(n=24)$ \\
\hline
\end{tabular}

"According to the definition that Gore et al. ${ }^{3)}$ proposed, the patients were classified into three groups including 'hyperlordotic', 'lordotic' and 'kyphotic'.

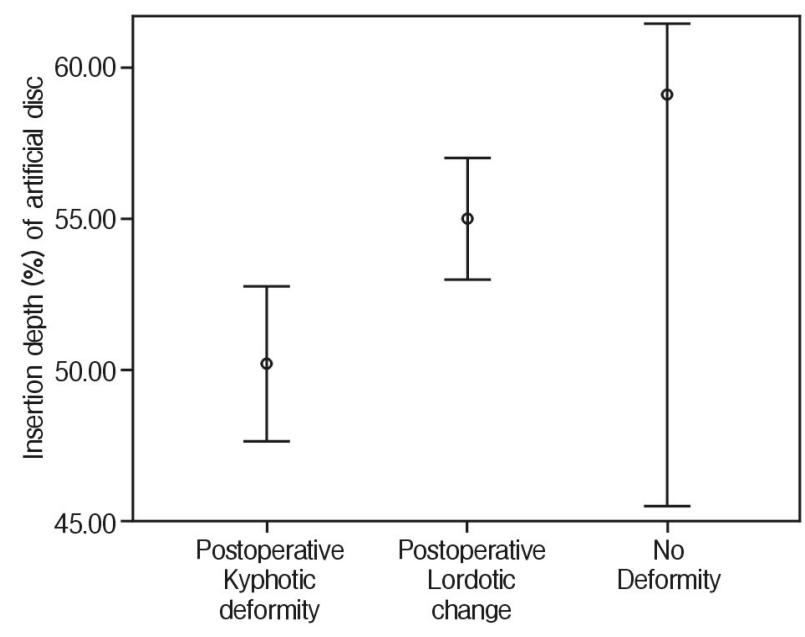

Fig. 4. Comparison of mean disc insertion depth between the group with postoperative kyphotic deformity, the group with postoperative lordotic change and the group with no deformity in segmental angle.

rest thirty-five patients was $54.26 \%$. So the result of analysis didn't show any difference between the group with postoperative kyphotic deformity and the other group without postoperative kyphotic deformity (Table 2).

Gore et al..$^{3)}$ proposed that a $16^{\circ}$ to $22^{\circ}$ cervical lordosis in men and a $15^{\circ}$ to $25^{\circ}$ cervical lordosis in women were normal. According to this definition, the patients were divided into three groups including 'hyperlordotic', 'lordotic' and 'kyphotic'. And we analyzed those who were hypothesized as postoperative kyphotic deformity to search any correlation with insertion depths of Prodisc-C artificial disc. But the group with postoperative kyphotic deformity didn't show any statistical significant difference in insertion depths of Prodisc-C artificial disc from other groups (Table 2).

According to the concept that decrease more than 5\% in postoperative C2-7 Cobb's angle is assumed as postoperative kyphotic deformity, twenty-six patients were classified as the group with postoperative kyphotic deformity. We analyzed them statistically to search any correlation with artificial disc insertion depth. But no significant difference was found from the others, either (Table 2).

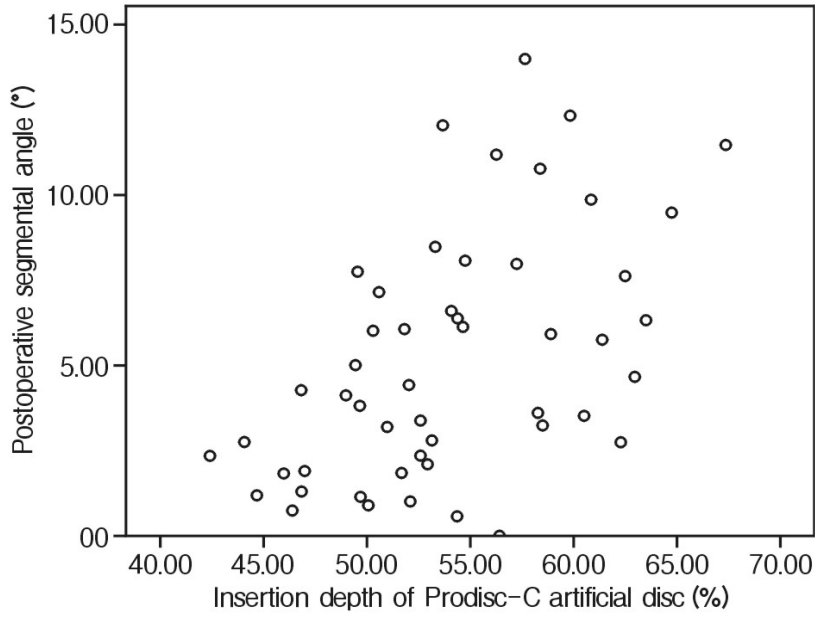

Fig. 5. Scatter diagram of artificial disc insertion depth and postoperative segmental angle $(R=0.516, p<0.001)$.

Finally, the patients were classified into three groups according to the changes between preoperative and postoperative segmental angles. Mean artificial disc insertion depth of those who showed postoperative kyphotic deformity in segmental angle was $50.18 \pm 4.23 \%$. And mean artificial disc insertion depth of those with postoperative lordotic change and those without postoperative deformity were $54.98 \pm 5.76 \%$ and $59.10 \pm 5.49 \%$, respectively. Mean artificial disc insertion depth of the group with postoperative kyphotic deformity was smaller than that of the group with postoperative lordotic change or the group without postoperative deformity $(\mathrm{p}<0.05$, Fig. 4). Positive correlation was found between artificial disc insertion depth and postoperative segmental angle $(\mathrm{R}=0.516$, $\mathrm{p}<0.001$, Fig. 5). These two results mean anterior located disc led to lordotic postoperative segmental angle and posterior located disc led to kyphotic segmental angle.

But the range of artificial disc insertion depth in the group with postoperative kyphotic deformity in segmental angle was not apart from that of the group with postoperative lordotic change (Fig. 4). The confidence interval of the group with postoperative kyphotic deformity in segmental angle was from 47.63 to $52.75 \%$, and that of the group with postoperative lordotic change was from 52.97 to $56.99 \%$. Technically these 
two confidence intervals don't overlap. But it failed to estimate proper insertion depths of Prodisc-C artificial disc effective in preventing postoperative kyphotic deformity.

\section{DISCUSSION}

As an alternative surgery to ACDF, TDR surgery has been an emerging procedure for cervical radiculopathy. Many authors have reported that TDR shows similar or better clinical outcomes $^{8,10)}$, or some advantages compared to $\mathrm{ACDF}^{1}$. However cervical TDR has several complications including postoperative kyphotic deformity. Xu et al. ${ }^{12)}$ reported $60 \%$ of their patients developed endplate kyphosis. Johnson et al. ${ }^{5)}$ found $80 \%$ of their single-level TDR patients with Bryan artificial disc developed endplate kyphosis.

Recently, several design limitations and contributing factors for postoperative kyphotic deformity were raised in the literature. Yoon et al. ${ }^{14)}$ reported that postoperative kyphoticdeformity after TDR with Bryan artificial disc was caused by several factors, including over-milling at the dorsal endplate, the insertion angle of Bryan artificial disc, structural absence of lordosis in the Bryan artificial disc, surgical procedure to remove the entire posterior longitudinal ligament, and pre-existing kyphosis. Cao et al. ${ }^{2)}$ reported over-milling or asymmetric milling of dorsal endplate influences on the disc insertion angle and leads to postoperative kyphotic deformity. Yi et al. ${ }^{13)}$ reported that toprevent postoperative kyphotic deformity after TDR with Bryan artificial disc, intentional modificationin disc insertion angle and depth would be helpful.

But Prodisc- $\mathrm{C}$ artificial disc is more sensitive to the surgical location of device than Bryan artificial disc, because Prodisc-C artificial disc has fixed center of rotation (COR) but Bryan artificial disc has variable COR. So Prodisc-C artificial disc needs to be confirmed about the correlation between the insertion depth of artificial disc and postoperative kyphotic deformity.

This study was focused to investigate the correlation between insertion depth of Prodisc-C artificial disc and postoperative kyphotic deformity, and the range of artificial disc insertion depth effective in preventing postoperative kyphotic deformity.

Postoperative segmental angle of operated level showed positive correlation with insertion depth ofProdisc-C artificial disc, but postoperative C2-7 angle didn't show any correlation with insertion depth of Prodisc-C artificial disc.

The group with postoperative segmental kyphosis had smaller insertion depth of Prodisc-C artificial disc compared to others. But when patients were classified into postoperative C2-7 kyphosis group according to several definitions of cer- vical kyphosis, none of them showed any statistical difference in insertion depth of Prodisc-C artificial disc. This result was similar to that of Yi et al. ${ }^{13)}$ reported.

There are some literatures that postoperative kyphotic deformity isn't associated with clinical outcomes ${ }^{\text {}}$. But once kyphosis has developed, axial loads tend to cause further kyphosis, thus initiating a vicious cycle and progression of the deformity. And cervical kyphosis tends to developan associated neurological deficit or chronic neck pain ${ }^{11}$ ). So postoperative kyphotic deformity is undesirable condition and should be avoided after TDR surgery.

There are some weaknesses in this study. First of all, other factors that may influence postoperative kyphotic deformity weren't controlled enough. For example, overmilling of dorsal endplate is one of the most important factors that can induce postoperative kyphotic deformity ${ }^{214}$. But as a rule, we keel the dorsal endplate before the insertion of Prodisc-C artificial disc, not mill. And the number of cases wasn't enough to subdivide according to the degrees of milling. So whether milling was performed or not and the degrees of milling weren't included as variables. This uncontrolled factor may lead to the unproven correlation of postoperative C2-7 kyphotic deformity and insertion depth of Prodisc-C artificial disc. And the definitions of normal cervical lordosis and postoperative kyphotic deformity need more research. Third, many patients have been lost in outpatient follow-up and the periods of evaluating postoperative kyphotic deformity are irregular. If postoperative kyphotic deformity proceeds in course of time after surgery, it needs to be controlled, too. Clinical outcomes after surgery weren't evaluated or analyzed according to the degree of postoperative kyphotic deformity.

\section{CONCLUSION}

Postoperative segmental kyphotic deformity was associatedwith insertion depth of Prodisc-C artificial disc. Anterior located artificial disc leads to lordotic segmental angle and posterior located artificial disc leads to kyphotic segmental angle postoperatively. But C2-7 Cobb's angle is not affected by artificial disc location after surgery. Further study with compensating weak points is required.

\section{REFERENCES}

1. Anderson PA, Rouleau JP: Intervertebral disc arthroplasty. Spine (Phila Pa 1976) 29:2779-2786, 2004

2. Cao JM, Zhang YZ, Shen Y, Xu JX, Ding WY, Yang DL, et al: Clinical and radiological outcomes of modified techniques in Bryan cervical disc arthroplasty. J Clin Neurosci 18:1308- 1312, 
2011

3. Gore DR, Sepic SB, Gardner GM: Roentgenographic findings of the cervical spine in asymptomatic people. Spine (Phila Pa 1976) 11:521-524, 1986

4. Heary RF, Karimi RJ: Evaluation, indications, and techniques of revision spine surgery in Youmans JR, Winn HR (eds): Youmans Neurological Surgery, ed 6. Philadelphia: Saunders/Elsevier, 2011, p2809

5. Johnson JP, Lauryssen C, Cambron HO, Pashman R, Regan JJ, Anand N, et al: Sagittal alignment and the Bryan cervical artificial disc. Neurosurg Focus 17:E14, 2004

6. Kaptain GJ, Simmons NE, Replogle RE, Pobereskin L: Incidence and outcome of kyphotic deformity following laminectomy for cervical spondylotic myelopathy. J Neurosurg 93:199-204, 2000

7. McAviney J, Schulz D, Bock R, Harrison DE, Holland B: Determining the relationship between cervical lordosis and neck complaints. J Manipulative Physiol Ther 28:187-193, 2005

8. Mummaneni PV, Burkus JK, Haid RW, Traynelis VC, Zdeblick TA: Clinical and radiographic analysis of cervical disc arthro- plasty compared with allograft fusion: a randomized controlled clinical trial. J Neurosurg Spine 6:198-209, 2007

9. Naderi S, Benzel EC, Baldwin NG: Cervical spondylotic myelopathy: surgical decision making. Neurosurg Focus 1: e1, 1996

10. Park JH, Roh KH, Cho JY, Ra YS, Rhim SC, Noh SW: Comparative analysis of cervical arthroplasty using mobi-c(r) and anterior cervical discectomy andfusion using the solis(r) -cage. J Korean Neurosurg Soc 44:217-221, 2008

11. Steinmetz MP, Kager CD, Benzel EC: Ventral correction of postsurgical cervical kyphosis. J Neurosurg 98:1-7, 2003

12. Xu JX, Zhang YZ, Shen Y, Ding WY: Effect of modified techniquesin Bryan cervical disc arthroplasty. Spine (Phila Pa 1976) 34:1012-1017, 2009

13. Yi S, Shin HC, Kim KN, Park HK, Jang IT, Yoon do H: Modified techniques to prevent sagittal imbalance after cervical arthroplasty. Spine (Phila Pa 1976) 32:1986-1991, 2007

14. Yoon DH, Yi S, Shin HC, Kim KN, Kim SH: Clinical and radiological results following cervical arthroplasty. Acta Neurochir (Wien) 148:943-950, 2006 